\title{
Three-Dimensional Statistical Gas Distribution Mapping in an Uncontrolled Indoor Environment
}

\author{
Matteo Reggente and Achim J. Lilienthal \\ AASS Research Center - Learning Systems Lab \\ Örebro University - Sweden \\ Email: matteo.reggente@oru.se,achim@lilienthals.de
}

\begin{abstract}
In this paper we present a statistical method to build three-dimensional gas distribution maps (3D-DM). The proposed mapping technique uses kernel extrapolation with a tri-variate Gaussian kernel that models the likelihood that a reading represents the concentration distribution at a distant location in the three dimensions. The method is evaluated using a mobile robot equipped with three "e-noses" mounted at different heights. Initial experiments in an uncontrolled indoor environment are presented and evaluated with respect to the ability of the 3D map, computed from the lower and upper nose, to predict the map from the middle nose.
\end{abstract}

Keywords: 3D-gas distribution, e-nose, gas sensing, mobile robots, kernel density estimation, model evaluation. PACS: $01.30 . \mathrm{Cc}$

\section{INTRODUCTION}

An increased quality of environmental monitoring is desired to protect the environment from toxic contaminants released into the air by vehicle emissions, power plants, refineries, to name but a few. Monitoring urban environments is typically done using immobile monitoring stations. Their total number and thus the number of sampling locations is limited by economical and practical constraints. Thus, the selection of monitoring/sampling locations becomes very critical, especially considering the time-varying, complicated local structure of the gas distribution. A further disadvantage of stationary air monitoring is that the monitoring stations are typically placed at expected "hot spots", close to busy roads, for example, and accordingly "background areas" are not monitored [1]. These issues can be addressed by mobile robots equipped with an "electronic nose", a combination we refer to as a mobile nose or "m-nose". An m-nose can act as a wireless node in a sensor network. With its selflocalization capability and the ability to adaptively select sampling locations, m-noses offer a number of important advantages, among others: monitoring with higher resolution, the possibility of source tracking, integration into existing application, compensation for inactive sensors, and adaption to dynamic changes in the environment. Using mobile robots for air quality monitoring is addressed in the EU project DustBot, for example, in which robot prototypes are developed to clean pedestrian areas and concurrently monitor the pollution levels [2].

Gas distribution modelling is the task of deriving a truthful representation of the observed gas distribution from a set of spatially and temporally distributed measurements of relevant variables, foremost gas concentra- tion (as used in this paper), but also pressure, and temperature, for example. Building gas distribution models is very challenging. One main reason is that in many realistic scenarios gas is dispersed chaotically by turbulent advection, resulting in a concentration field that consists of fluctuating, intermittent patches of high concentration [3]. In principle, CFD (Computational Fluid Dynamics) models can be applied, which try to solve the governing set of equations numerically. However, CFD models are computationally very expensive. They become intractable for sufficiently high resolution in typical real world settings and depend sensitively on accurate knowledge of the state of the environment, which is not available in practical situations. Here, we instead opt an alternative approach to gas distribution modelling and create a statistical model of the observed gas distribution, treating gas sensor measurements as random variables. Our approach creates a statistical model discretized to a grid map and it is "parameter-free" in the sense that it makes no assumptions about a particular functional form of the gas distribution. Previous approaches to statistical gas distribution mapping with mobile robots were largely restricted to mapping a $2 \mathrm{D}$ slice, parallel to the floor and level with the gas sensors on the robot (Sec. 2). The major contribution of this paper is the extension of Kernel extrapolation distribution mapping to three dimensions and its evaluation based on real world experiments in an uncontrolled indoor environment. This is an important step for gas distribution modelling since the gas distribution structure is essentially three dimensional. After a discussion of related work in the next section and the description of the hardware and set-up used for the monitoring trials (Sec. 3), we outline the 3D distribution mapping 
algorithm in (Sec. 4). Finally, we present first results and end with conclusions and suggestions for future work.

\section{RELATED WORK}

In urban environments, especially in areas with high population and traffic density, human exposure to hazardous substances is often significantly increased. High pollution levels exceeding air quality standards, have been observed in street canyons [4], for example. In a natural environment advective flow generally dominates gas dispersal compared to slow molecular diffusion. Since the airflow is almost always turbulent, the gas distribution becomes patchy and meandering [5]. As pointed out in the review of Vardoulakis et al. [4], just a few approaches to environmental monitoring with immobile sensing stations consider the complicated local structure of gas distribution. Acknowledging the need to refine the monitoring scale, Maruo et al. developed small inexpensive gas sensors for air pollution monitoring [6]. Addison et al. [7] propose a method for predicting the spatial pollutant distribution in a street canyon based on a stochastic Lagrangian particle model superimposed on a known velocity and turbulence field.

Gas distribution mapping with mobile gas sensors has been implemented and investigated by mobile robots equipped with an "e-nose" $[8,9,10,11,12]$. These approaches can be divided into two groups. Model-based approaches such as the one proposed by Ishida et al. [8] assume a particular model of the time-averaged gas distribution and estimate the corresponding parameters. Model- or parameter-free approaches deal with the fluctuating nature of the gas distribution either by recording individual concentration samples over a prolonged time (several minutes) $[11,12]$ or by statistically integrating subsequent measurements into a spatial grid $[9,10]$.

All the above mentioned approaches produce $2 \mathrm{D}$ gas distribution maps. In the field of mobile robot olfaction, the three-dimensionality of the environment is only taken into account in a few publications on gas source tracing $[13,14,15]$. Three-dimensional gas distribution mapping with a mobile robot has not been investigated before to the best of our knowledge.

\section{EXPERIMENTAL SET-UP}

An ATRV-JR robot equipped with a SICK LMS 200 laser range scanner (for localization) and three "electronic noses" was used for the monitoring experiments. The "electronic noses" comprise different Figaro 26xx gas sensors enclosed in an aluminum tube. These tubes are horizontally mounted at the front side of the robot at a height of $10 \mathrm{~cm}, 60 \mathrm{~cm}$ and $110 \mathrm{~cm}$ and actively ventilated through a fan that creates a constant airflow towards the gas sensors. This lowers the effect of external airflow or the movement of the robot on the sensor response and guarantees continuous exchange of gas in situations with very low external airflow. In this work, we address the problem of modeling the distribution from a single gas source. With respect to this task, the response of the different sensors in the electronic nose is highly redundant and thus it is suffcient to consider the response of a single sensor (TGS 2620) only.

The scenario selected for the gas distribution mapping experiments is to monitor an area of approx. $10 \times 3 \mathrm{~m}^{2}$ in a long corridor with open ends and a high ceiling. This choice was motivated by the goal to monitor uncontrolled environments and even pedestrian areas. During our monitoring trials there was disturbance caused by people passing by and by the opening of doors and windows. The gas source was a small cup filled with ethanol or acetone. This source was placed roughly in the middle of the investigated corridor segment at a height of $1.6 \mathrm{~m}$ to prevent the robot from colliding with the source and ensure a substantially 3D gas distribution with the chosen analytes (which are heavier than air: ethanol $\approx$ $46 \mathrm{~g} / \mathrm{mol}$ and acetone $\approx 58 \mathrm{~g} / \mathrm{mol}$ ). As a possible monitoring strategy, the robot followed either a random walk trajectory or a predefined sweeping path to cover the area of interest, using a fixed starting point.

In order to be able to relate the readings of the different electronic noses to each other, we perform a simple calibration by determining the baseline (response to clean air) and the maximum response in the actual experimental enviroment (but not in a controlled set-up) with the three e-noses positioned very close to each other. This is done by recording the respective minimum values $R_{\min }^{n}$ (baseline) and the maximum values $R_{\max }^{n}$ after a cup filled with the analyte was opened close to the noses. In the subsequent monitoring trial the raw readings $R_{i}^{n}$ from nose $n$ are scaled as

$$
r_{i}=\frac{R_{i}^{n}-R_{\min }^{n}}{R_{\max }^{n}-R_{\min }^{n}} .
$$

Thus, we make the assumptions that each sensor was exposed to the same minimum and maximum concentration during the calibration process and that the sensors' response depends on the concentration in the same, monotonous way. Since the calibration is repeatedly carried out in the same environment and under the same conditions as the actual experimental runs, we avoid issues with long-term drift and mitigate drift issues due to different temperature and humidity in the trials.

\section{3D GAS DISTRIBUTION MODEL}

In this section we introduce the basic ideas of the 3D Kernel GDM algorithm extending the 2D model of Lilienthal et al. [16], and describe briefly the Kernel DM+V algorithm [16] that models the distributions mean and the corresponding variance. The gas distribution mapping problem addressed here is to learn a predictive three dimen- 
sional model $p\left(r \mid \mathbf{x}, \mathbf{x}_{1: \mathbf{n}}, \mathbf{r}_{1: n}\right)$ for the gas concentration $r$ at location $\mathbf{x}$, given the robot trajectory $\mathbf{x}_{\mathbf{1}: \mathbf{n}}$ and the corresponding concentration measurements $\mathbf{r}_{\mathbf{1}: \mathbf{n}}$. We consider the case of a single target gas, but in principle the proposed method can be extended to the case of multiple different odor sources as described in [17]. We also assume perfect knowledge about the position $\mathbf{x}_{\mathbf{i}}$ of a sensor at the time of the measurement. To account for the uncertainty about the sensor position, the method in [18] could be used. To study how gas distribution in three dimensions we consider the concentration readings from multiple "e-noses" mounted at different heights. The central idea of kernel extrapolation methods is to understand gas distribution mapping as a density estimation problem that involves convolution with a kernel.The first step in the Kernel $\mathrm{DM}+\mathrm{V}$ algorithm is the computation of the weights $\omega_{i}^{(k)}$, which represent the importance of each sensors measurement $i$ at grid cell $k$ :

$$
\omega_{i}^{(k)}\left(\sigma_{x}, \sigma_{y}, \sigma_{z}\right)=\mathscr{N}\left(\left|x_{i}-x^{(k)}\right|, \sigma_{x}, \sigma_{y}, \sigma_{z}\right) .
$$

The weights are computed using a multivariate 3DGaussian kernel $\mathscr{N}$ evaluated at the distance between the location of the measurement $x_{i}$ and the center $x^{(k)}$ of cell $k$. We use a diagonal covariance matrix $\Sigma$ with elements $\sigma_{x}, \sigma_{y}, \sigma_{z}$, which defines the kernel extension along the three axis. Using Eq. 2, weights $\omega_{i}^{(k)}$, weighted sensor readings $\omega_{i}^{(k)} \cdot r_{i}$ and weighted variance contribution $\omega_{i}^{(k)} \cdot \tau_{i}$ are integrated and stored in temporary grid maps:

$$
\begin{aligned}
\Omega^{(k)}=\sum_{i=1}^{n} \omega_{i}^{(k)}, \quad R^{(k)} & =\sum_{i=1}^{n} \omega_{i}^{(k)} \cdot r_{i}, \quad V^{(k)}=\sum_{i=1}^{n} \omega_{i}^{(k)} \cdot \tau_{i}, \\
\tau_{i} & =\left(r_{i}-r^{k(i)}\right)^{2} .
\end{aligned}
$$

$\tau_{i}$ is the variance contribution of reading $i$ and $r^{k(i)}$ is the model prediction from the cell $k(i)$ closest to the measurement point $x_{i}$. From the integrated weight map $\Omega^{(k)}$ we compute a confidence map $\alpha^{(k)}$, which indicates high confidence for cells if the estimate can be based on a large number of readings recorded close to the center of the respective grid cell:

$$
\alpha^{(k)}\left(\sigma_{x}, \sigma_{y}, \sigma_{z}\right)=1-e^{-\frac{(\Omega)^{(k)}\left(\sigma_{x}, \sigma_{y}, \sigma_{z}\right)}{\sigma_{\Omega}^{2}}}
$$

$\sigma_{\Omega}$ is a scaling parameter that defines a soft margin which decides rather the estimate for a cell has high confidence or low confidence. By normalizing the map of weighted readings $R^{(k)}$ to $\Omega^{(k)}$ and linear blending with the best guess for the case of low confidence, we finally obtain the map estimates of the mean $r^{(k)}$ and the corresponding variance map $v^{(k)}$ as

$$
r^{(k)}\left(\sigma_{x}, \sigma_{y}, \sigma_{z}\right)=\alpha^{(k)} \frac{R^{(k)}}{\Omega^{(k)}}+\left\{1-\alpha^{(k)}\right\} \bar{r},
$$

$$
v^{(k)}\left(\sigma_{x}, \sigma_{y}, \sigma_{z}\right)=\alpha^{(k)} \frac{R^{(k)}}{\Omega^{(k)}}+\left\{1-\alpha^{(k)}\right\} v_{t o t} .
$$

The second terms in the equations are the best estimate for cells with a low confidence. $\bar{r}$ represents an estimate of the mean concentration for cells for which we do not have sufficient information from nearby readings, indicated by a low value of $\alpha(k)$. We set $\bar{r}$ to be the average over all sensor readings. The estimate $v_{t o t}$ of the distribution variance in regions far from measurement points is computed as the average over all variance contributions. The 3D Kernel-GDMV algorithm depends on seven parameters: the kernel widths $\sigma_{x}, \sigma_{y}, \sigma_{z}$, that govern the amount of extrapolation on individual readings according to three axis and the cell sizes $c_{x}, c_{y}, c_{z}$ that determines the resolution at which different predictions can be made and $\sigma_{\Omega}$.

\section{RESULTS}

Qualitative Comparison: In order to evaluate how well the model captures the true properties of the gas distribution we use two of the three noses (the lower and the upper one, see Sec. 3), to build a 3D model using the method described in the previous section. After that we slice the model and extract the layer corresponding to the height of the remaining middle nose ("3D@60cm"). From the readings of the middle nose we also build a 2D gas distribution map ("2D@60cm") and we compare it with the slice extracted from the 3D model. This evaluation method is visualized in Fig. 1. The first two maps at the top represent the mean distribution according to the models “2D@60cm" and “3D@60cm” obtained in a random walk experiment. These two maps display a structural similarity especially when comparing the highconcentration regions colour-coded in red. The two maps at the bottom of Fig. 1 represent the mean distribution obtained from the 2D models computed for the upper ("2D@110cm") and the lower nose (“2D@10cm").

Quantitative Comparison: As a measure of distribution similarity we use the Kullback-Leibler (KL) divergence or relative entropy [19] for probability functions:

$$
K L(p \mid q)=-\int p(x) \ln \frac{q(x)}{p(x)} d x
$$

where $p(x)$ is the "unknown" distribution (in our case "2D@60cm"), and $q(x)$ is the modelled distribution (“3D@60cm”). Since the gas distributions maps are not probability distribution we first normalize them so that the sum over all values equals to one. Then we compute the KL divergence for 14 layers of the 3D model for two different experiments, one with an ethanol source and one with an acetone source. As can be seen in Fig. 2, the minimum of the KL divergence was found exactly for the layer at the height of the middle nose in both experiments. 


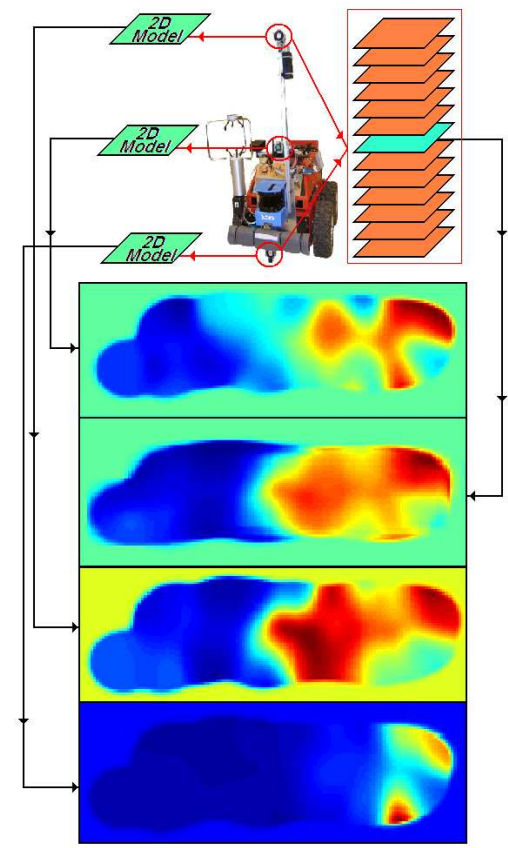

FIGURE 1. Top to bottom: picture of the "m-nose" prototype ("Rasmus") carrying the three electronic noses; mean of the 2D gas distribution map obtained from the middle nose (“2D@60cm”); from slicing the 3D model (“3D@60cm”) obtained from the lower and the upper nose; 2D mean map from the upper nose (" $2 \mathrm{D} @ 110 \mathrm{~cm}$ "); from the lower nose (“2D@10cm”).

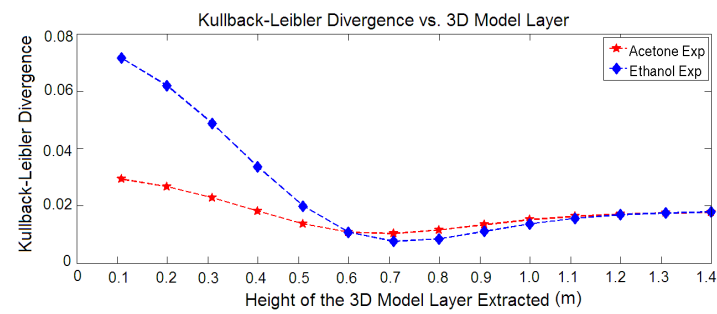

FIGURE 2. KL divergence for a random walk experiment with ethanol (blue line) and a sweeping experiment with acetone (red line) between 14 layers of the 3D model and the “2D@60cm”.

\section{DISCUSSION AND CONCLUSIONS}

3D gas distribution modelling with a mobile robot in an uncontrolled environment is a challenging field of research. This is mainly due to the chaotic nature of the dispersed gas. Utilization of mobile robots to monitor pollution has a number of advantages reflected by an increasing interest in this field in the last ten years. In this paper we present a statistical method to build threedimensional gas distribution maps (3D-DM). The mapping technique uses Kernel extrapolation mapping with a tri-variate Gaussian weighting function to model the decreasing likelihood that a reading represents the true concentration with respect to the distance in the three dimensions. The method is evaluated using a mobile robot equipped with three "e-noses" mounted at different heights. Initial experiments in an uncontrolled indoor enviroment are presented and evaluated with respect to the ability of the 3D map, computed from the lower and upper nose, to predict the map from the middle nose. This paper represents initial work and of course more trials are needed in different environments and with different analytes. Another interesting task is to integrate wind measurements, obtained by an anemometer to build an improved gas distribution model.

\section{REFERENCES}

1. K. Kemp, and F. Palmgren, Annual Report, NERI, Roskilde pp. 155-182 (1999).

2. DustBot - Networked and Cooperating Robots for Urban Hygiene, http://www.dustbot.org (2006-2009).

3. B. Shraiman, and E. Siggia, Nature pp. 639-646 (2000).

4. S. Vardoulakis et al., Atmospheric Environment 37, 155-182 (2003).

5. P. Roberts, and D. Webster, "Turbulent Diffusion," in Environmental Fluid Mechanics - Theories and Application, 2002.

6. Y. Maruo et al., Atmospheric Environment 37, 1065-1074 (2003).

7. P. Addison et al., Environmental Monitoring and Assessment pp. 333-342 (2000).

8. H. Ishida et al., Sensors and Actuators B 49 (1998).

9. A. Hayes et al., IEEE Sensors Journal, Special Issue on Electronic Nose Technologies 2, 260-273 (2002).

10. A. Lilienthal, and T. Duckett, Robotics and Autonomous Systems 48, 3-16 (2004).

11. A. H. Purnamadjaja, and R. A. Russell, "Congregation Behaviour in a Robot Swarm Using Pheromone Communication," in Proc. ACRA, 2005.

12. P. Pyk et al., Auton Robot 20, 197-213 (2006).

13. H. Ishida et al., "Three-Dimensional Gas/Odor Plume Tracking with Blimp," in Proc. ICEE, 2004.

14. A. Rutkowski et al., "A Robotic Platform for Testing Moth-Inspired Plume Tracking Strategies," in Proc.ICRA, 2004.

15. H. Ishida.et al., "Three-Dimensional Gas-Plume Tracking Using Gas Sensors and Ultrasonic Anemometer," in IEEE Sensors (2004), 2004, pp. 1175-1178.

16. A. J. Lilienthal et al., "A Statistical Approach to Gas Distribution Modelling with Mobile Robots - The Kernel DM+V Algorithm," in submitted to IROS, 2009.

17. A. Loutfi et al., Robotica p. online (2008).

18. A. J. Lilienthal et al., "A Rao-Blackwellisation Approach to GDM-SLAM, Integrating SLAM and Gas Distribution Mapping," in (ECMR), 2007, pp. 126-131.

19. S. Kullback, and R. A. Leibler, Annals of Mathematical Statistics 2, 79-86 (1951). 